\title{
The use of active IR thermography and wavelet analysis for non-destructive evaluation
}

\author{
by L. Rozanski* and K. Ziopaja*
}

\author{
*Poznan University of Technology, 60-965 Poznań, ul. Piotrowo 2, Poland, leszek.rozanski@gmail.com
}

\begin{abstract}
In the paper, the possibility of detection the hidden defects or damage applying the models of dynamic (active) infrared thermography and the 2D discrete wavelet transform of data measured in thermal processes has been described. The possibility to examine the defects of the material structure with the discrete wavelet transform (DWT) of the spatial function registering the disturbance underwent analysis. Besides of theoretical information about application of these transforms, the results of MES digital simulations as well as qualitative experimental have been presented. The performed digital simulations proved that the 2D discrete wavelet transform could be effective tool for non-destructive identification of the structural defects. High level of the noise compared to the disturbances generated by the structural defect causes some limitations of this method.
\end{abstract}

\section{Introduction}

Nowadays, to solve the scientific and technical problems, it is necessary to develop and to improve the methods and apparatus of non-destructive testing and inspection. In the industrial enterprises, this is stimulated by wide application of the in-process control systems aimed to ensure high quality of the products. As a result, the scientists and engineers are looking for new more effective methods of non-destructive inspection. One of the most important methods is an infrared dynamical (active) thermography. There are three basic methods of dynamical thermography described in the literature: pulse thermography (PT), pulse phase thermography (PPT), and lock-in thermography referred to as a modulated thermography (MT). There are several kinds of the modulated thermography [1]. Methods of dynamical thermography enable to generate a parametrical images. Mathematical apparatus applied for that purpose contains methods of Fourier's transform as well as the wavelet transform. The comparison of those methods application in the detection of defects is presented in the [2]. In both cases, the argument of the transformed functions is the time. In the paper, the possibility to examine the defects of the material structure with the discrete wavelet transform of the spatial function registering the disturbance underwent analysis.

\section{Model of the heat flow transient state}

The equations describing the heat flow transient state in the area $\Omega$ are described with Fourier's low as following:

$$
\left.\begin{array}{c}
-\operatorname{div} \mathbf{q}(\mathbf{x}, t)+f=c(\mathbf{x}) \dot{T}(\mathbf{x}, t) \\
\mathbf{q}(\mathbf{x}, t)=-\lambda(\mathbf{x}) \cdot \nabla T(\mathbf{x}, t)
\end{array}\right\} \text { in } \Omega
$$

Symbols $\mathbf{q}, f, \lambda, T$ correspond with heat flow, heat generated in the volume unit, matrix of the heat conductivity and the temperature field, respectively. The symbol $\dot{T}$ derivate, and $c$ is specific heat. Convection coefficient $h$ was determined for the assumption of no transport of the air mass around area $\Omega$. Conditions of the heat transfer through outer surfaces $\Gamma_{T}, \Gamma_{q}, \Gamma_{h}$ are described with the appropriate boundary conditions: Dirichlet's, Neumann's and Henkel's (2):

$$
\left\{\begin{array}{cc}
T(\mathbf{x}, t)=T^{0}(\mathbf{x}, t) & \text { na } \Gamma_{T} \\
q_{n}(\mathbf{x}, t)=q_{n}^{0}(\mathbf{x}, t) & \text { na } \Gamma_{q} \\
q_{n}(\mathbf{x}, t)=h\left[T(\mathbf{x}, t)-T_{\infty}(\mathbf{x}, t)\right] & \text { na } \Gamma_{h}
\end{array}\right.
$$

In case of the heat flow transient state, the initial condition was assumed $T(\mathbf{x}, t=0)=T_{0}$, and the environment temperature $T_{\infty}(\mathbf{x}, t)=22^{\circ} \mathrm{C}$. 


\section{Wavelet transform as a tool for signal analysis}

Theoretical background for the practical applications of the wavelet analysis was published in the early 1990s by Daubechies, Chui and Newland [3], [4],[5]. Mallat [6] presented the algorithm of multi-distributive decomposition of twodimensional signal $F(x, y) \in L^{2}(R)$ in the wavelet representation:

$$
F(x, y)=S_{J}(x, y)+\sum_{j=1}^{J} D_{j}^{V}(x, y)+\sum_{j=1}^{J} D_{j}^{H}(x, y)+\sum_{j=1}^{J} D_{j}^{D}(x, y),
$$

where the functions $S_{J}, D_{j}^{V}, D_{j}^{H}$ and $D_{j}^{D}$ correspond with the smoothened part of the image, and with vertical, horizontal and diagonal detail of the image. Maximal level of the discrete wavelet transform $J$ depends on the number of points $N$ used for approximation of the function $F(x, y) ; N=2^{J}$. Representation of the signal on the level $j$ is the sum of vertical, horizontal and diagonal components:

$$
D_{j}(x, y)=D_{j}^{V}(x, y)+D_{j}^{H}(x, y)+D_{j}^{D}(x, y) \text {. }
$$

The detail $D_{j=1}(x, y)$ is corresponding to the most local representation of the function $F(x, y)$.

\section{Digital simulations and measurement results}

Figure 1a illustrates the analysis of the defects detection effectiveness for the aluminium plate, heated from the left side. In that case, heat flow transient state was simulated, where the heat transfer took place along the longer side of the plate. Thermal input function is described by the Drichlet condition, and its model is shown in the Fig. 1a. Digital simulation if the heat flow transient state, taking into consideration the natural convection $h=6.25\left[\mathrm{~W} /\left(\mathrm{m}^{2} \mathrm{~K}\right)\right]$, has been performed with the program Abaqus Standard. To carry out discretization of the plate, the spatial, 8-junction linear finite elements DC3D8 were applied, distributed steadily. Figure 1a illustrates the analysis of the defect detection effectiveness for the aluminium plate, heated from the left side. In that case, heat flow transient state was simulated, where the heat transfer took place along the longer side of the plate. Thermal input function is described by the Dirichlet condition, and its model is shown in the Fig. $1 \mathrm{~b}$.

a)

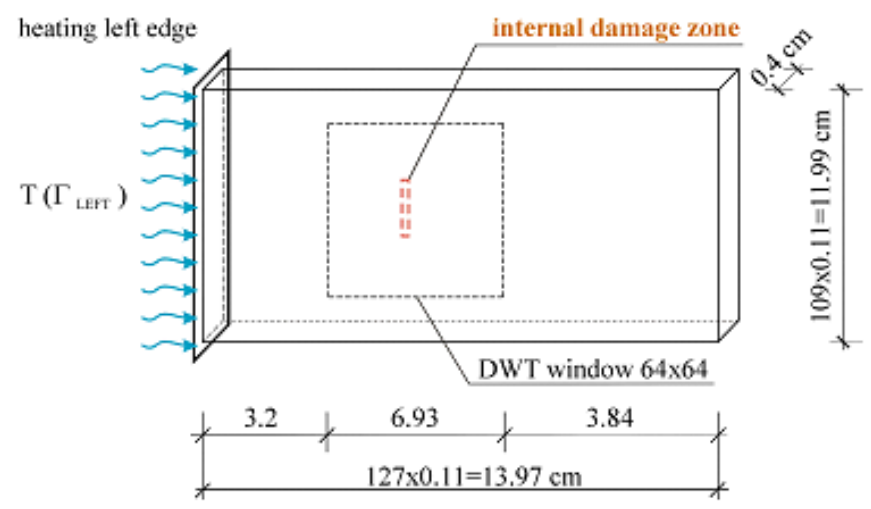

b)

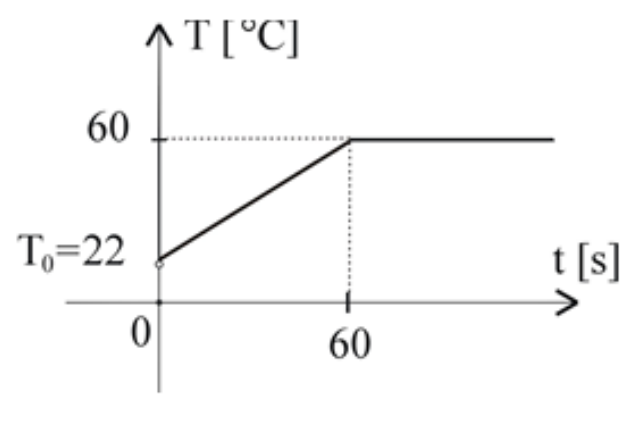

Fig. 1. Aluminium plate heated from the left side; a) model of the system, b) model of the thermal input

The following parameters were assumed for the simulation: thermal conductivity $\lambda=250 \mathrm{~W} /(\mathrm{m} \cdot \mathrm{K})$, specific heat $c=900 \mathrm{~J} /(\mathrm{kg} \cdot \mathrm{K})$ and bulk density $\gamma=2700 \mathrm{~kg} / \mathrm{m}^{3}$. The defect was modulated as a cut $0.2 \mathrm{~cm}$ wide and $2.0 \mathrm{~cm}$ long. Its depth was simulated unsteadily $g=(0.08,0.16,0.24$, and $0.32 \mathrm{~cm})$. The temperature distribution was modelled on the opposite side to the defected one. In the measuring window of $64 \times 64$ joints, the registered values of temperature underwent the discreet wavelet transformation. The result of simulation led to several important conclusion. First of all, it revealed that the thermal process improves the possibility of the defect localization when the temperature registration time is longer $(t=$ $60 \mathrm{~s}$ ). On the other hand, for depth $g=0.08$ and $0.16 \mathrm{~cm}$, it is almost impossible to detect the defect. At the edges of the 
transform, there appear strong disturbances (Figure 2) caused by the finite dimensions of the analyzed temperature field, as well as by "internal" procedures of the wavelet transformation.

a)

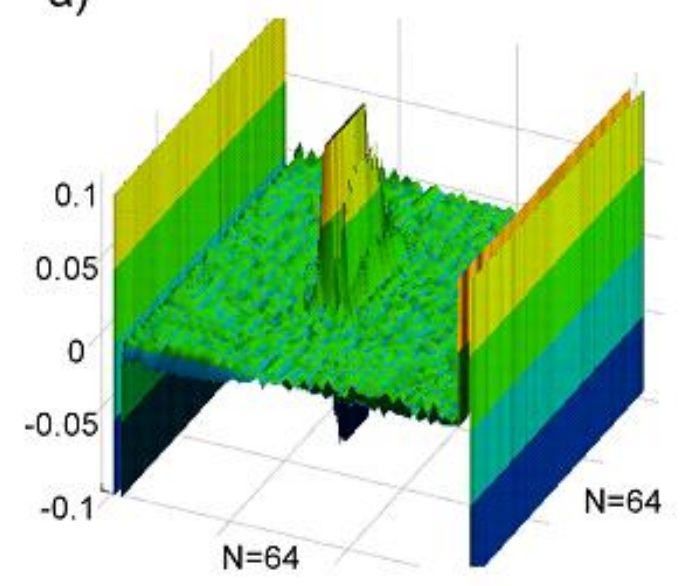

b)

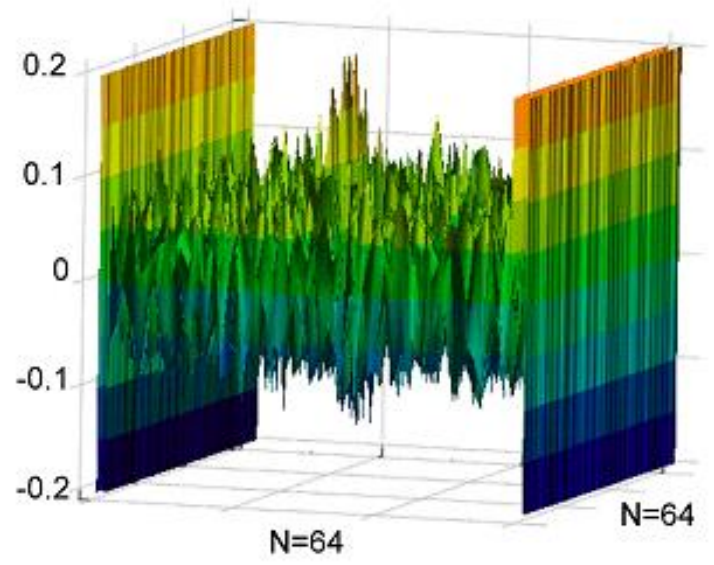

Fig. 2. Wavelet transformation of the temperature field (T64x64), Detial D1, Daubechies 4, t=60 s: a) depth of cut $\mathrm{g}=0.32 \mathrm{~cm}$; b) depth cut $\mathrm{g}=0.32 \mathrm{~cm}$, white noise $\pm 0,1^{\circ} \mathrm{C}$

It should be noted, however, that the presented example proved the ability of defect detection despite of the noise which is almost present in the real conditions of measurement. The laboratory measurement was performed using the plate dimensions $140 \times 140 \times 4 \mathrm{~mm}$. Fig. 3a presents the results of the experiment for registration time $t=47 \mathrm{~s}$. In the Fig. $3 \mathrm{~b}$, the defect is cleary seen in the graph of the transform of the temperature field.

a)

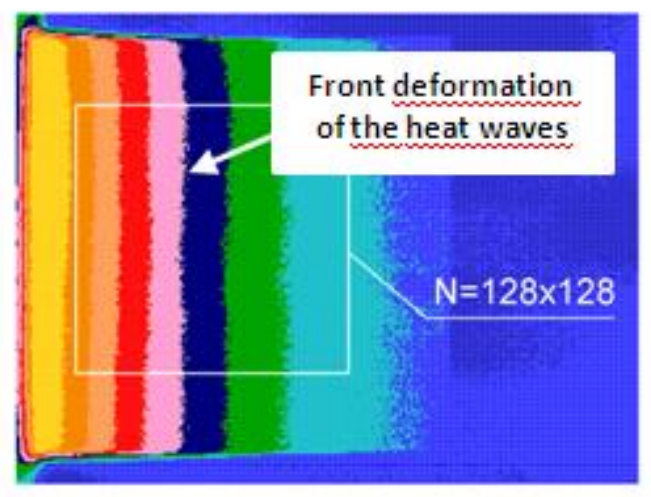

b)

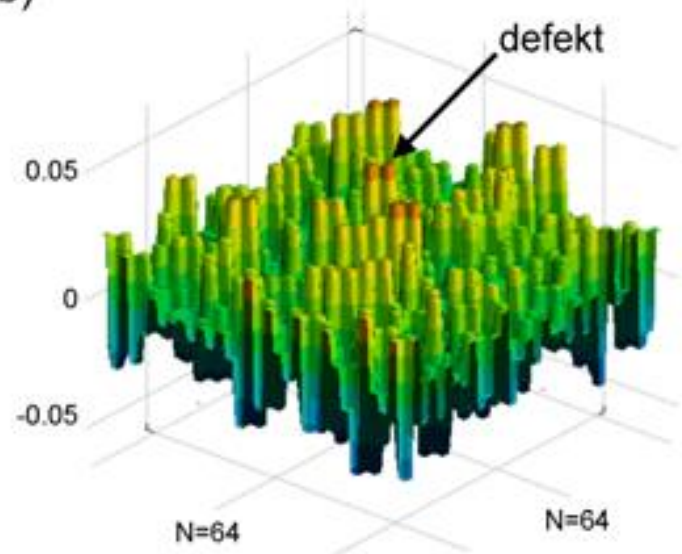

Fig. 3. The results of experimental research:

a) Thermogram for $t=47 \mathrm{~s}$, b) Transform of the temperature field

The thermal process behaved in the similar way as it was described in the digital example. The temperature field was registered with the long-wave thermographic camera FLIR SC 2000 (Fig. 4). Its thermal resolution defined in NETD terms is $0.1{ }^{\circ} \mathrm{C}$ for the temperature value $30{ }^{\circ} \mathrm{C}$. and nominal accuracy is $\pm 2{ }^{\circ} \mathrm{C}$ The object's defect was a cut with dimensions $2 \times 0.2 \mathrm{~cm}$ and depth $0.25 \mathrm{~cm}$. Because of the noise, it is not possible to determine exact localization of the defect directly from the graph of the temperature field transform. 


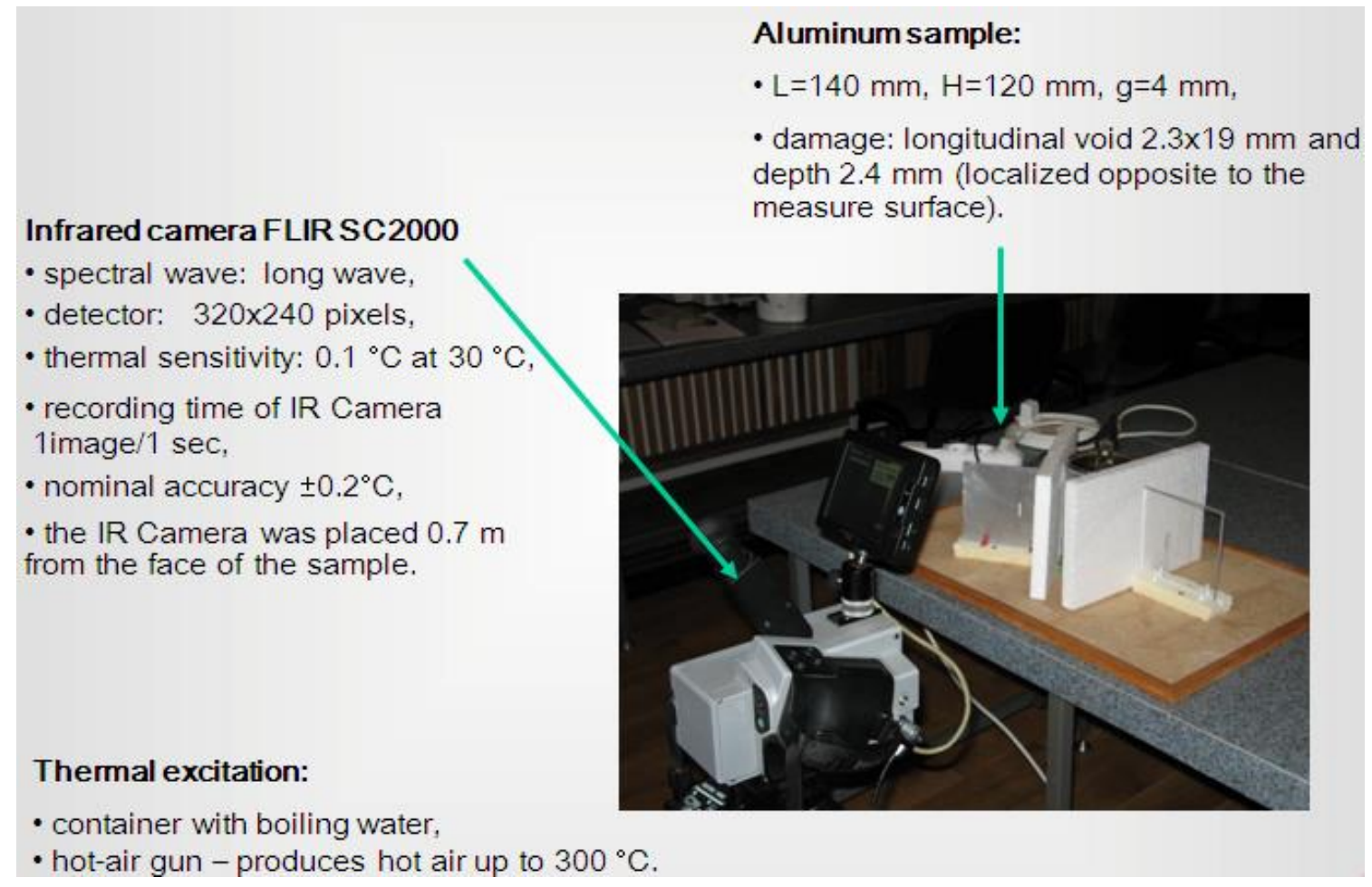

Fig. 4. Measurement set-up for active thermography

\section{Conclusion}

The performed digital simulation proved that the 2D discrete wavelet transform could be an effective tool for nondestructive identification of the structural defects. High level of noise compared to the disturbances generated by the structural defect causes some limitations of this method. In case of transverse heat flux problem the measurement errors can play an important role. In wavelet transform boundary disturbance can appear (in plane heat flux). It creates difficulties in damage identification. However, the effectiveness could be improved when thermographic system of higher resolution is applied. The other way to improve the effectiveness is to repeat the registration of the thermal field and to perform the average procedure to get less noisy thermal images. Discrete wavelet transform is a powerful tool in damage detection but sufficient accuracy of experiments must be ensured.

\section{REFERENCES}

[1] Carlomango G. M. , C. Meola C., "Comparison between thermographic techniques for Frescoes NDT". NDT\&E International, 35, pp 559-565, 2002.

[2] R. Olbrycht R. et al., "Comparison of Fourier and wavelet analyses for defect detection in lock-in and pulse thermography” QIRT Journal, No 2, pp 219-232, 2007.

[3] I. Daubechies, "The wavelet transform, time-frequency localization and signal analysis". IEEE Transactions on Information Theory, Vol. 36, No 5, pp 961-1005

[4] Chui Ch. K., 'An Introduction to Wavelets" Academic Press, London, 1992.

[5] D. E. Newland, "An introduction to random vibrations, spectral and wavelet analysis", Wiley, New York, 1993.

[6] S. Mallat, 'A theory for multiresolution signal decomposition: the wavelet representation', IEEE Transactions on Pattern Analysis and Machine Intelligence, Vol. 11, No 7, pp 674-693, 1989. 